Research Article

\title{
Prevalence of Escherichia Coli and Its Antimicrobial Susceptibility Profiles among Patients with UTI at Mulago Hospital, Kampala, Uganda
}

\author{
Isaac Odongo, Ronald Ssemambo, and Joseph M. Kungu (iD) \\ College of Veterinary Medicine, Animal Resources and Biosecurity Makerere University, P. O. Box 7062, Kampala, Uganda \\ Correspondence should be addressed to Joseph M. Kungu; kungu@live.com
}

Received 18 October 2019; Revised 13 December 2019; Accepted 14 January 2020; Published 1 February 2020

Academic Editor: Subhada Prasad Pani

Copyright ( 2020 Isaac Odongo et al. This is an open access article distributed under the Creative Commons Attribution License, which permits unrestricted use, distribution, and reproduction in any medium, provided the original work is properly cited.

\begin{abstract}
Background. Urinary tract infections (UTIs) remain the most common infections diagnosed in in- and outpatients as well as hospitalized patients. Current knowledge on antimicrobial susceptibility pattern for uropathogens is essential to effectively manage UTIs. This study aimed at determining the prevalence of $E$. coli and its antimicrobial susceptibility profiles among patients presenting with signs and symptoms of UTI in Mulago Hospital in Uganda. Methods. Midstream urine samples were collected from 100 patients presenting with signs and symptoms of UTI at the outpatient department of Mulago Hospital. The samples were cultured, and isolates of $E$. coli (predominant bacteria) are subjected to sensitivity testing against most commonly used antibiotics. Results. Out of 100 patients studied, E. coli was the most dominant with a prevalence of $10 \%$. The prevalence was high among females $(11.5 \%)$ compared with males $(8.3 \%)$ and among the age group of $\leq 17$ years. Escherichia coli isolates were highly susceptible to cefotaxime/clavulanic acid (100\%) and nitrofurantoin (70\%) but showed high resistance to cefuroxime (100\%), ceftazidime (100\%), nalidixic acid (90\%), and ciprofloxacin (90\%). Conclusion. Escherichia coli, the predominant uropathogen, showed significant multidrug resistance to antibiotics commonly prescribed for the management of UTIs. These findings should form a basis for preliminary decision making on the appropriate line of treatment for UTIs.
\end{abstract}

\section{Background}

Urinary tract infections (UTIs) are among the most common bacterial infections encountered in primary health care, and it is among the most common infections with an increasing resistance to antimicrobial agents [1]. These infections have also become the most common hospital-acquired infections, accounting for up to $35 \%$ of nosocomial infections and second cause of bacteremia in hospitalized patients. This ailment affects patients in all age groups and sexes, with females accounting for $87.5 \%$ of the cases compared with males (71.3\%) [2-4]. This is associated with the short urethral tube of women and close proximity of the anus to the urethral opening, allowing easier access of the bacteria to the urethra. It is estimated that half of all women will have recurrent episodes of acute cystitis during adult life [5].

The enterobacterales order is the most common etiological agent of urinary tract infections because they have several factors associated with their attachment to the uroepithelium such as possession of adhesins [6]. Escherichia coli has been documented to be the most common pathogen associated with urinary tract infections in many countries causing both community- and hospital-acquired UTI $[7,8]$. Other pathogens such as Enterococcus sp., Staphylococcus saprophyticus ("honeymoon cystitis"), Klebsiella sp., Enterobacter sp., Citrobacter sp., and ProteusMorganella-Providencia sp. may also occur [9].

The emergence and spread of antibiotic-resistant pathogens is a major public health threat. Resistant pathogens especially enterobacteriaceae can withstand lethal doses of antibiotics with various chemical structures and mechanisms of action [10]. Enterobacteriaceae such as E. coli exhibit important mechanisms to avoid lethal doses of drugs such as aminoglycoside degrading enzyme, target alteration, decreased uptake, beta lactamase enzyme degradation, and overexpression of efflux proteins. The rate of drug discovery 
and its development in the 21st century cannot match with the continuous and detrimental change in antibiotic resistance trends [11]. Since the introduction of fluoroquinolones in 1960s in an attempt to optimise nalidixic acid, there has been no broad-spectrum antibiotics discovered and developed. Pathogens associated with UTI are increasingly changing their features particularly due to self-medication, overuse, and misuse of drugs [12]. With the rampant antimicrobial misuse, there is a rise in the newer and more resistant strains of the etiological agents of UTI. Bacterial infections resistant to antibiotics can limit effective treatment rendering bacterial infections difficult to treat including UTI. In low-income countries, there is a reduced access to health care and high cost of second line drugs which limits the use of newer broad-spectrum drugs [2].

The resistance patterns of community-acquired UTI have not been studied exclusively, yet knowledge regarding common uropathogens and their susceptibility patterns to drugs is key in improving prescription decisions [12]. Treatment of patients at Mulago hospital is usually carried out empirically and regular laboratory culture and sensitivity tests of urine from UTI patients are limited [13].

\section{Methods}

2.1. Study Design. A cross-sectional study was conducted from January to March 2018, to determine the prevalence and antimicrobial susceptibility profiles of $E$. coli among patients presenting with UTI at the outpatient's department of Mulago National Referral Hospital, Kampala, Uganda.

All patients with signs and symptoms of UTI who voluntarily consented were recruited in the study. Participants who qualified for the study after signing the consent forms provided midstream urine samples, and information about their age and sex was gathered. The urine samples were cultured to determine the presence of E. coli organisms. Positive samples for $E$. coli were further subjected to antimicrobial susceptibility testing to evaluate their antimicrobial-susceptible profiles.

2.2. Sample Size Determination. The sample size was determined using a formula by Thrusfield as follows [14]:

$$
n=\frac{z^{2} p(1-p)}{i^{2}}
$$

where $n$ is the calculated sample size, $z$ is the desired level of confidence (1.96), $i$ is the standard sampling error (10\%), and $p$ is the estimated prevalence 50\% [15]. Although a minimum sample size required was 96 , up to 100 patients from Mulago Hospital who met the inclusion criteria were recruited in the study to increase precision.

2.3. Collection and Analysis of Urine Samples. A total of 100 midstream urine samples were collected into a sterile urine container on the same day of enrollment. The samples were sent to the laboratory for analysis, and most of the samples were analyzed within one hour after collection.

$10 \mu \mathrm{l}$ of well-mixed urine samples were inoculated on MacConkey agar (Oxoid limited, United Kingdom) using a sterile loop following standard culture procedures. The plates were incubated at $37^{\circ} \mathrm{C}$ for 24 hours. Morphological colony identification and biochemical tests were used to confirm the E. coli organisms. Disc diffusion method was used to determine the antibiotic susceptibility of E. coli. Escherichia coli isolates were suspended in peptone water and incubated at $37^{\circ} \mathrm{C}$ until turbid and turbidity adjusted to a standard uniform concentration of $0.5 \mathrm{McFarland}$ solutions. The isolates were then inoculated on Mueller Hinton agar (Oxoid, United Kingdom). The antibiotic discs containing precise concentration of the antibiotics were individually placed $1 \mathrm{~cm}$ from the wall and from each other. The plates were then incubated at $37^{\circ} \mathrm{C}$ for 24 hours. The diameter zones of clearance were measured in millimetres and interpreted according to the Clinical Laboratory Standard Institute (CLSI) protocol [16]. For quality control, E. coli ATCC 25922 provided in the laboratory was used as a control strain.

2.4. Data Analysis. The data from the study were entered and cleaned in MS Excel and analyzed using SPSS software version 20. The Chi-squared test was used to perfoFrm descriptive statistics at $95 \%$ level of confidence (Table 1).

\section{Results}

3.1. Prevalence of Escherichia coli and Other Bacteria among UTI Patients. Out of the 100 samples collected, E. coli was the most dominant pathogen at $10 \%$. There were other isolates identified in the urine samples which included; $C$. freundi (2\%), K. pneumoniae (2\%), S. aureus (1\%), P. aeruginosa (1\%), and Streptococcus sp. (1\%). More females (52\%) participated in the study compared with the males (48\%). The prevalence of $E$. coli was higher among the females (6\%) compared with the males (4\%). Furthermore, the prevalence was also higher in the age group of 0-17 years (4\%) compared with other groups as described in Table 2.

Cross tabulations of demographic variables (age and sex) with prevalence of E. coli, using Chi-squared test was performed at $95 \%$ level of confidence. There were no significant differences between the variables and E. coli prevalence.

3.2. Antimicrobial Susceptibility of E. coli Isolates to the Common Antibiotics. All the E. coli isolates were sensitive cefotaxime/clavulanic acid (100\%). Most of the isolates were sensitive to nitrofurantoin (70\%), and few showed sensitivity to nalidixic acid and ciprofloxacin (10\%). However, all the isolates showed significantly high sensitivity to cefuroxime and ceftazidime (100\%). The results of the pattern of antibiotic susceptibility are shown in Table 3 .

\section{Discussion}

Up to date information on prevalence and antibiotic susceptibility pattern of pathogenic bacteria is essential in 
Table 1: Prevalence of E. coli and other bacteria among UTI patients in Mulago Hospital.

\begin{tabular}{lcc}
\hline Isolate & Number of isolates & Percent \\
\hline E. coli & 10 & 10 \\
C. freundi & 2 & 2 \\
K. pneumoniae & 2 & 2 \\
Streptococcus spp & 1 & 1 \\
P. aeruginosa & 1 & 1 \\
S. aureus & 1 & 1 \\
\hline
\end{tabular}

TABLE 2: Cross tabulation of demographic variables with prevalence of E. coli.

\begin{tabular}{|c|c|c|c|c|c|c|}
\hline Variable & Category & E. $\operatorname{coli}(+)$ & E. $\operatorname{coli}(-)$ & Prevalence & $X^{2}$ & $P$ value \\
\hline \multirow{2}{*}{ Sex } & Male & 4 & 44 & 8.3 & 0.285 & 0.594 \\
\hline & Female & 6 & 46 & 11.5 & & \\
\hline \multirow{4}{*}{ Age groups in years } & $0-17$ & 4 & 31 & 11.4 & 0.635 & 0.88 \\
\hline & $18-30$ & 2 & 28 & 6.7 & & \\
\hline & $31-50$ & 2 & 18 & 10 & & \\
\hline & $>51$ & 2 & 13 & 13.3 & & \\
\hline
\end{tabular}

Table 3: Antibiotic susceptibility findings of the E. coli isolated from urine samples of UTI patients in Mulago Hospital.

\begin{tabular}{|c|c|c|c|}
\hline Antibiotic & Concentration of antibiotic in the disc $(\mu \mathrm{g})$ & Resistant & Susceptible \\
\hline Ciprofloxacin & 5 & $9(90.0 \%)$ & $1(10.0 \%)$ \\
\hline Ceftazidime & 30 & $10(100 \%)$ & $0(0.0 \%)$ \\
\hline Cefotaxime/clavulanic acid & $30 / 10$ & $0(0.0 \%)$ & $10(100 \%)$ \\
\hline Nalidixic acid & 30 & $9(90.0 \%)$ & $1(10.0 \%)$ \\
\hline Nitrofurantoin & 300 & $3(30.0 \%)$ & $7(70.0 \%)$ \\
\hline Cefuroxime & 30 & $10(100 \%)$ & $0(0.0 \%)$ \\
\hline
\end{tabular}

therapeutic management of UTIs. This study established that prevalence of E. coli among UTI patients was fairly low (10\%) compared with other related studies. In a previous study conducted in patients attending hospitals in Bushenyi district western Uganda by Odoki and others, a significantly high prevalence of $E$. coli $41.9 \%$ was reported [13]. Likewise, previous related studies in Mulago Hospital showed a higher prevalence of 57.5 and $50 \%$, respectively [15, 17]. The prevalence of this study was low compared with the previous possibly due to population variation and significant differences in the sample sizes. It is also possible that with time, tremendous improvements in management of UTIs and community hygiene could have contributed to reduction of the prevalence of E. coli organisms [15].

The prevalence was clearly high in females $(11.5 \%)$ compared with the males $(8.3 \%)$ though it was not statistically significant. A number of previous studies also documented the prevalence of E. coli in UTI patients to be high in females compared with males $[2,6,13,18,19]$. This could be due to the close proximity of anus to the warm urethral tube. Furthermore, the urethral tube of the females is short, and this shortens the distance moved by the organism to the bladder. These predisposing factors of UTI are accelerated by limited resources, poor hygiene, and low socioeconomic status [2]. Alteration in the vaginal microflora significantly play an important role in encouraging colonization of the vagina with coliforms, and this can be associated with UTI [20].
The prevalence was revealed to be high in the age group of $\leq 17$ years, $11.4 \%$, compared with other age groups though it was not statistically significant. The findings are similar to the findings reported in Nigeria [21]. The juveniles have low immunity against infections and could never have been exposed to the infections before, and this might have highly exposed them to the infections. Furthermore, young children in most cases in our setting are misdiagnosed since symptoms of UTI in infants resemble the symptoms of other infections such as fever, vomiting, and refusing to eat. Those in adolescence age are involved in increased sexual activity which predisposes them to UTI $[13,21]$.

Escherichia coli isolated showed a high sensitivity of $100 \%$ to cefotaxime/clavulanic acid. This could be due to the fact the drug combinations are not easily resisted by the organism. The clavulanic acid targets the enzyme beta lactamase that is responsible for resistance against beta lactam antibiotics, and this helps the drug to overcome resistance; combination of cephalosporins and beta lactamase inhibitor are among the first new drug formulations that may possess clinically relevant broad-spectrum antibacterial activity. Furthermore, it has been rarely used in the treatment of urinary tract infections and other infections and therefore present organisms present low resistance to it since they have not been frequently exposed to it $[1,6,22]$.

The results from the study also showed that $70 \%$ of the isolates were susceptible to nitrofurantoin. An earlier study in the same study setting involving nonpregnant women had 
shown high sensitivity of E. coli to nitrofurantoin at $100 \%$ sensitivity rates [17]. Likewise, another study reported a similar susceptibility of 78\% [15]. This shows a decline in the sensitivity rates of $E$. coli to this antibiotic. The increasing resistance could be due to increased overuse and misuse of antibiotics. The persistor cells (defined as metabolically inactive cells that neither grow or die when exposed to bactericidal concentrations of the antibiotics) presents another important challenge as these cells tend to be associated with treatment failure, recurrence, and chronic infections as they continue to replicate after the antibiotic therapy is discontinued [23]. It could also possibly due to the cheap costs and ready availability of this drug since previous Uganda Clinical Guidelines 2016 recommended it in the empirical treatment of urinary tract infections [13].

Escherichia coli isolates were highly resistant to cefuroxime and ceftazidime, and $100 \%$ was so alarming in this study. The resistance in a previous study in Mulago Hospital was significantly lower [15]. Resistance to nalidixic acid and ciprofloxacin was also alarming (90\%), and this was similar to a related study in Mulago which reported $89.9 \%$ resistance of E. coli [17]. Ciprofloxacin has always been used in the empirical treatment of urinary tract infections. This resistance could be due to previously increased use of these drugs since the previous Uganda Clinical Guidelines 2010 recommended its use in the empirical treatment of UTI, and also these drugs are relatively cheaper and readily available. This could have rendered them easily accessible to the patients, increasing their misuse and overuse, leading to resistance.

\section{Conclusion}

Escherichia coli, the predominant organism, observed in the UTI patients was low. There was a significantly high resistance to cefuroxime, ceftazidime, nalidixic acid, and ciprofloxacin to E. coli isolated. Continuous use of these drugs might most likely be associated with treatment failure and serious antimicrobial resistance. There was acceptably high sensitivity to cefotaxime/clavulanic acid and nitrofurantoin. This study recommends that urine culture and sensitivity should be done when UTI is strongly suspected to guide clinicians and physicians in treatment decisions. The study recommends that either nitrofurantoin and cefotaxime-clavulanic acid or their combination be used in the empirical treatment of UTI in the Ugandan settings. There should be continuous periodic antibiotic resistance monitoring to curb resistance emergence.

\section{Abbreviations}

CLSI: Clinical Laboratory Standard Institute Protocol (CLSI)

UTI: Urinary Tract Infections.

\section{Data Availability}

The data used to support the findings of this study are included within the article.

\section{Ethical Approval}

Research ethical approval was sought and granted from Mulago Hospital Research and Ethics Committee (reference number: MHREC 1563).

\section{Consent}

Formal consent was obtained from every participant before being enrolled.

\section{Conflicts of Interest}

The authors declare that they have no conflicts of interest.

\section{Authors' Contributions}

IO conceptualized and designed the study; collected, analyzed, and interpreted the data; drafted and critically reviewed the manuscript; and gave final approval for submission of manuscript. RS collected the data, critically reviewed the manuscript, and gave final approval for submission of manuscript. JMK conceptualized and designed the study, critically reviewed the manuscript, and gave final approval for submission of manuscript. All authors have read and approved the manuscript.

\section{Acknowledgments}

The authors would like to acknowledge all the participants for accepting to take part in the study. The authors also thank Joselyn Nansereko, Sharifa Kasinga, Donum Nakabuye, Winfred Namee, Joy, and Leymond Kalema for their support during the study.

\section{References}

[1] A. Bryce, A. D. Hay, I. F. Lane, H. V. Thornton, M. Wootton, and C. Costelloe, "Global prevalence of antibiotic resistance in paediatric urinary tract infections caused by Escherichia coli and association with routine use of antibiotics in primary care: systematic review and meta-analysis," BMJ, vol. 352, no. i939, 2016.

[2] M. Akram, M. Shahid, and A. U. Khan, "Etiology and antibiotic resistance patterns of community-acquired urinary tract infections in J N M C Hospital Aligarh, India," Annals of Clinical Microbiology and Antimicrobials, vol. 6, no. 1, p. 4, 2007.

[3] K. Gupta, D. F. Sahm, D. Mayfield, and W. E. Stamm, "Antimicrobial resistance among uropathogens that cause community-acquired urinary tract infections in women: a nationwide analysis," Clinical Infectious Diseases, vol. 33, no. 1, pp. 89-94, 2001.

[4] M. Gajdács, K. Burián, and G. Terhes, "Resistance levels and epidemiology of non-fermenting gram-negative bacteria in urinary tract infections of inpatients and outpatients (RENFUTI): a 10-year epidemiological snapshot," Antibiotics, vol. 8, no. 3, p. 143, 2019.

[5] L. E. Nicolle, "Urinary tract pathogens in complicated infection and in elderly individuals," The Journal of Infectious Diseases, vol. 183, no. s1, pp. S5-S8, 2001. 
[6] F. S. Nas, M. Ali, M. S. Abdallah, and A. U. Zage, "Prevalence and antibiotic susceptibility pattern of Escherichia coli isolated from urine samples of urinary tract infection patients," $A R C$ Journal of Urology, vol. 4, no. 1, 2019.

[7] S. Sabir, A. A. Anjum, T. Ijaz, M. A. Ali, M. U. Rehman, and M. Nawaz, "Isolation and antibiotic susceptibility of E. coli from urinary tract infections in a tertiary care hospital," Pakistan Journal of Medical Sciences, vol. 30, no. 2, pp. 389-392, 1969.

[8] M. Gajdács, M. Ábrók, A. Lázár, and K. Burián, “Comparative epidemiology and resistance trends of common urinary pathogens in a tertiary-care hospital: a 10-year surveillance study," Medicina, vol. 55, no. 7, 2019.

[9] M. Gajdács and E. Urbán, "Resistance trends and epidemiology of citrobacter-enterobacter-serratia in urinary tract infections of inpatients and outpatients (RECESUTI): a 10year survey," Medicina, vol. 55, no. 6, pp. 1-13, 2019.

[10] M. Gajdács, "The concept of an ideal antibiotic: implications for drug design," Molecules, vol. 24, no. 5, 2019.

[11] E. Medina and D. H. Pieper, "Tackling threats and future problems of multidrug-resistant bacteria," Current Topics in Microbiology and Immunology, vol. 398, pp. 3-33, 2016.

[12] N. Kaur, M. Shweta, and H. C. Madan, "Urinary tract infection: aetiology and antimicrobial resistance pattern in infants from a tertiary care hospital in North India," Journal of Clinical and Diagnostic Research, vol. 8, no. 10, pp. DC01DC03, 2014.

[13] M. Odoki, A. A. Aliero, J. Tibyangye et al., "Prevalence of bacterial urinary tract infections and associated factors among patients attending hospitals in Bushenyi district, Uganda," International Journal of Microbiology, vol. 2019, Article ID 4246780, 8 pages, 2019.

[14] M. Thrusfield, Veterinary Epidemiology, Blackwell Publishing, Hoboken, NJ, USA, 3rd edition, 2007.

[15] D. Kabugo, S. Kizito, D. D. Ashok et al., "Factors associated with community-acquired urinary tract infections among adults attending assessment centre, Mulago Hospital Uganda," African Health Sciences, vol. 16, no. 4, pp. 11311142, 2016.

[16] C. Institute, Performance Standards for Antimicrobial Susceptibility Testing. CLSI supplement M100S, Clinical and Laboratory Standards Institute, Wayne, PA, USA, 26th edition, 2016.

[17] A. D. Mwaka, H. Mayanja-Kizza, E. Kigonya, and D. KadduMulindwa, "Bacteriuria among adult non-pregnant women attending Mulago hospital assessment centre in Uganda," African Health Sciences, vol. 11, no. 2, pp. 182-189, 2011.

[18] I. O. Okonko, L. A. Ijandipe, O. A. Ilusanya et al., "Incidence of urinary tract infection (UTI) among pregnant women in Ibadan, South-Western Nigeria," African Journal of Biotechnology, vol. 8, no. 23, pp. 6649-6657, 2009.

[19] R. M. Mordi and P. O. Erah, "Susceptibility of common urinary isolates to the commonly used antibiotics in a tertiary hospital in southern Nigeria," African Journal of Biotechnology, vol. 5, 2006.

[20] T. M. Hooton and W. E. Stamm, "Diagnosis and treatment of uncomplicated urinary tract infection," Infectious Disease Clinics of North America, vol. 11, no. 3, pp. 551-581, 1997.

[21] O. A. Aiyegoro, S. Africa, E. E. Odjadjare, E. O. Igbinosa, and A. I. Okoh, "Incidence of urinary tract infections (UTI) among children and adolescents in Ile-Ife, Nigeria," African Journal of Microbiology Research, vol. 11, no. 3, pp. 13-19, 2007.

[22] G. Schmiemann, I. Gágyor, E. Hummers-Pradier, and J. Bleidorn, "Resistance profiles of urinary tract infections in general practice-an observational study," BMC Urology, vol. 12, no. 1, 2012.

[23] B. Van den Bergh, M. Fauvart, and J. Michiels, "Formation, physiology, ecology, evolution and clinical importance of bacterial persisters," FEMS Microbiology Reviews, vol. 41, no. 3, pp. 219-251, 2017. 